\title{
Construction Safety and Occupational Health Education in Egypt, the EU, and US Firms
}

\author{
Amal ElSafty ${ }^{1}$, Adel ElSafty ${ }^{2}$, Maged Malek ${ }^{3}$ \\ ${ }^{1}$ Department of Occupational \& Environmental Medicine, Cairo University, Egypt \\ ${ }^{2}$ School of Engineering, University of North Florida, Jacksonville, USA \\ ${ }^{3}$ Construction Management Department, University of North Florida, Jacksonville, USA \\ Email: amal_safty@yahoo.com, adel.el-safty@unf.edu,mmalek@unf.edu
}

Received June 29, 2012; revised July 30, 2012; accepted August 15, 2012

\begin{abstract}
The construction industry has been considered an accident prone industry. Alarming statistics indicate that the construction industry accounts for 55,000 fatal injuries each year. That is because construction sites are often filled with potential hazards that can lead to serious injury or death. Responsibility for these injuries may be attributed to a variety of individuals including owners, contractors or subcontractors, engineers, equipment manufacturers, and architects. This mandated a new safety culture to emerge resulting in a decline in the incidence rate of all recordable nonfatal injury and illness for construction workers. This study explores the safety culture encountered in two large construction companies in the USA and Egypt, over the last five years. Comparison of the results recorded in both companies was also conducted. The study also investigated the benefits of focusing on the occupational health in the construction field in Egypt and USA. In addition to the literature review, the research methodology used for this study was inspired by the Contextual Design (CD) Methodology. The study indicated the positive impact of implementing safety programs on significantly reducing the recordable injuries. It also showed the benefits of focusing on occupational health and wellness, training, and education of workers.
\end{abstract}

Keywords: Occupational Health; Workplace Safety; Accidents; Construction Industry

\section{Introduction}

The construction work site is usually a chaotic place with incredibly high activities. The accident rates closely correlate to the level of activity within the industry, indicating that when work load is high, safety tends to receive less attention. The dangers faced by construction workers are alarming. The rate of death of workers is higher in construction industry than any other industry. Moreover, construction industry results in a high rate of death by injury. Although construction represents only $6 \%$ of US workers, it produces $20 \%$ of the fatalities.

The Occupational Safety and Health Act of 1970 defines occupational safety and health standard as, "a standard which requires conditions, or the adoption or use of one or more practices, means, methods, operations, or processes, reasonably necessary or appropriate to provide safe or healthful employment and places of employment" [1-3]. According to the Bureau of Labor Statistics (BLS) reports $[4,5]$, the U.S. construction industry workers suffered more than 1,200 fatal occupational injuries. In 2008, the BLS report indicated a slight decrease in injury rate to be 5.4 cases per 100 full-time workers; yet these jobs remain inherently high-risk. Alarming statistics indicate that the construction industry accounts for 55,000 fatal injuries each year; meaning one person is killed in a site accident every ten minutes [6]. Construction hazards may include falls, extreme heights, falling from rooftops, machinery failure, unguarded machinery, being struck by heavy construction equipment, electrocutions, silica dust, asbestos, lead, welding emissions, accidents, structure collapses, roofing and pavement tar, engine exhaust fumes, and other hazards. Construction sites include residential construction, bridge erection, roadway paving, excavations, demolitions, and big painting jobs.

Construction safety education is a vital part of the building trades. The construction agenda in the US was presented in National Occupational Research Agenda (NORA) [7]. The National Safety Council presents the significant advantages of safety to employers showing the higher profitability to their businesses by reducing accidents rates and maintaining high productivity. Safety training is mandatory for all construction workers. The work culture should encourage workers to participate in safety education programs. These programs must test the ability to recognize workplace hazard, the awareness of emergency plan, the ability to fill the safety check list, 
the implementation of safe procedures, the demonstration of professional attitude and maturity level. Maintaining the ability of workers to cope with physical and psychosocial demands at work becomes increasingly important in prolonging working life.

In Egypt, the construction sector is a major contributor to the country's economy and one of its fastest-growing sectors. This growth is estimated at an average of $20 \%$ to $22 \%$ annually since the 1980 s. It is fueled by the continuously increasing demand for housing and by the country's large infrastructure projects. It is undoubtedly important to improve construction safety and to give due attention to occupational injuries and illnesses, recording system, and prevention methods, and developing new skills for safety management.

The construction industry puts the workers under great strain and exposes them to many potential health risks. There are also a number of accidents that can happen in the Egyptian construction industry due to many aspects like faulty and damaged equipment. At the first sign of wear and tear, the equipment should stop being used for safety reasons. Other common risks in Egypt include scaffolding collapse, falls from ladders, safety harnesses breaking, and fall from a roof. A research shows that the major causes of accidents are related to the unique nature of the industry, human behavior, difficult work site conditions, and poor work site management which results in unsafe work methods, equipment and procedure [8]. It is important to define who is responsible for what, as follows:

- A responsibility of the Health and Safety Executive (HSE) is to enforce safety regulations.

- The employer should ensure the safety of the workplace. He should also address ergonomic issues. The Occupational Safety and Health Administration (OSHA) requires employers to keep records of illnesses and injuries that occur in the workplace. It also requires them to provide the Bureau of Labor Statistics certain data on work-related injuries and illnesses for the purpose of year-to-year monitoring. A case is considered recordable if it is new, work-related and meets one or more of the general reporting requirements, as determined by OSHA.

- The employee is responsible for reporting any defects in the workplace or on any of the equipment that is being used. A workplace inspection is a critical part of a comprehensive safety and health program in which the workplace is examined closely on a regular basis for the purpose of:

- Identifying and recording potential and actual hazards associated with buildings, equipment, environment, and processes.

- Identifying hazards which require immediate attention.
- Ensuring that existing hazard controls are functioning and recommending corrective action.

- OSHA has a role to assure safe and healthy conditions for workers by enforcing the standards developed under the Act. That could be done by encouraging the States in their efforts to assure safe and healthful working conditions; by providing research, information, education, and training in the field of occupational safety and health.

- Personal Safety records will provide employers with reliable OSHA certified data about workers' safety.

- The Severe Violator Enforcement Program (SVEP) directive became effective on June 18, 2010, to better focus enforcement efforts by concentrating inspection resources on employers who have demonstrated resistance or indifference to their OSH Act obligations by committing willful, repeated, or failure-to-abate violations in one or more of the following circumstances: 1) a fatality or catastrophe situation; 2) in industry operations or processes that expose employees to the most severe occupational hazards and those identified as "High-Emphasis Hazards"; 3) exposing employees to hazards related to the potential release of a highly hazardous chemical; or 4) all egregious enforcement actions.

OSHA has also identified some violations/citations that include:

- A willful violation as one committed with intentional disregard of, or plain indifference to, requirements of the Occupational Safety and Health Act and regulations.

- A serious citation is issued when death or serious physical harm result from a hazard about which the employer knew, or should have known.

- A repeat violation occurs when a company has been cited for similar violations within the past three years.

In the absence of the correct health and safety in construction sites, accidents will continue to occur and can result in severe injuries. Such injuries may include spinal cord damage, head and neck injury, broken/fractured bones, torn muscles/ligaments, and back injury. It is worth noting that injuries can result in loss in productiveity and high cost of workers' compensation and healthcare. In an effort to avoid such losses, periodic evaluations of hazard exposure levels in the field are implemented as part of the occupational health risk prevention. Also, in order to ensure the health of construction workers, a more proactive occupational health standard is needed. For the occupational health of construction workers, regular examinations, blood tests, x-rays, and medical evaluations should be established.

\section{Research Design, Methodology, and Approach}




\subsection{Aim of Work}

The recent surge in construction accidents and the inefficiency of the recording system in Egypt prompted the authors to review the safety standards in this sector. This study was designed to assess the state of the safety performance, practices, and techniques of large construction projects (large projects were defined as those having construction costs in excess of $\$ 10$ million). The study team aims at exploring the safety culture within the Egyptian construction industry to provide important insights into how companies operating in Egypt manage their safety programs in construction sites. Also the team compared the health and safety measures as well as prevalence of incidents encountered in different construction work places located in Egypt and USA. One of our main objectives was to improve safety management in construction sites, leading progressively to the enhancement of construction safety performances.

\subsection{Information for Selected Egyptian and US Firms}

US firm; Gate Precast is considered to be one of the nation's largest manufacturers of architectural and structural precast concrete systems. Gate Precast is operating eight manufacturing facilities. The facilities are located in Alabama, Kentucky, North Carolina, Tennessee, two in Florida, and two in Texas. At Gate's state-of-the art plants, architectural and structural concrete are designed, manufactured, delivered and installed to customers throughout the United States. Gate is very responsible in its efforts to respect the environment, community, the health and safety of the employees.

Egyptian firm; Modern Construction is one of the construction companies that perform construction projects of commercial and residential buildings, airports, roadways and highway pavement, architectural facades, and structural concrete and steel construction. The company offers full-service engineering to its clients throughout four provinces in Egypt. The services include structural, mechanical, electrical, plumbing, and architectural services. It employs 120 engineers, architects, technicians, and construction workers.

\subsection{Methodology \& Approach}

The methodology followed in this study is summarized in the following steps:

1) Literature review to collect the basic knowledge essential for survey development.

2) Research design to develop a safety investigation Performa to collect data from studied construction worksites. These firms have implemented the basic Five High-Impact Zero Accidents Techniques (safety pre-task/ pre-project planning, orientation training, safety incentives, alcohol and substance abuse program, and accident investigations). The developed Performa covered a wide range of safety practices and techniques. These questions included all levels of personnel associated with each project. The specific research inquiries include:

- Existing hazards/environmental factors to which workers may be exposed.

- Past incidents, injuries, illnesses, fatalities.

- Workplace air levels of lead, manganese, cadmium, chromium and iron.

- Types of worker injuries/illnesses/conditions specific to construction and welding.

- Recent safeguards implemented to enhance worker safety.

- Plans for future safety implementations.

Information about the health and safety measures, conduction of educational safety training courses, use of protective equipment, records of recordable injuries in the last three years, and days away from work were all investigated. Recording workplace injuries and illnesses were documented in a locally developed Performa at the specific workplace. An OSHA recordable injury is an occupational injury or illness that requires medical treatment more than simple first aid and must be reported. Also, the OSHA Recordable Criteria include death, loss of consciousness, days away from work, restricted work, job transfers, significant injuries or illnesses diagnosed by physician such as cancer, asbestosis, fractures or punctured eardrums, and medical treatment beyond first aid.

3) Categorization of recordable injuries were performed by considering the "Days Away, Restricted or Transferred" (DART) and Lost Work Days. A DART case is a subset of OSHA recordable cases where the injury/illness is severe enough that the individual loses time away from his/her job by being away from work, on restricted duty, or is transferred to another job function because of the injury. For analysis of worksite injuries and fatalities, OSHA collects many forms of data which helps aid its analysis of worksite injuries and fatalities. There are different types of graphs; among which are the total recordable incident rate (TRIR), days away recorded/transferred (DART), and lost work day incident rate (LWDI). The average recordable rate was computed for each of the participating projects. This was defined as the average number of recordable injuries per 200,000 hours of worker hours of exposure. The values represented above can be calculated using this general equation: \{"Variable" RATEx (200,000 hours/annual hours worked) $\}$, where the "Variable" in the equation refers to the rate that you are trying to find. The value of 200,000 comes from doing a survey of 100 workers, where each worker works 2000 hours per year on average [1-3]. 
- TRIR represents the number of occurring incidents including "near miss" incidents.

- DART represents the recorded number of days workers are away from work (injury related). This is useful when analyzing total production rate and how much loss is incurred from injuries.

DART rate $=$ DART cases $\times(200,000$ hours/annual hours worked)

- LWDI represents the workday injuries that occur. This is useful for interpreting solutions to lowering the number of injuries and fatalities per year.

4) Data were collected through personal interviews conducted with key safety personnel at the job sites. Interviews were conducted in a time frame that ranged from three to four hours.

5) Analysis of data was performed. Recommendations and conclusions were drawn based on the analysis of the data.

6) Also, the research methodology used for this study was inspired by the Contextual Design (CD) Methodology $[9,10]$. The first step of CD is CI, in which construction workers were interviewed on construction sites. The CI unveiled information about their work practices, the tools and equipment used to conduct work activities, the workflow, and existing training methods. Interviews were administered and consisted of observation, questionnaires and assessment of the user environment as well as, a safety assessment of the design if considered as potentially affecting the personnel safety. Data collected from interviews were interpreted and work models were figured out in context of construction companies. The work models were consolidated further to gain a holistic understanding of construction companies. The second and third steps involved interpreting data and building individual work description from each interview, followed by consolidated work models. The different work models helped the designers understand the similarities, variations, roles, responsibilities, restrictions, workflows, and structures of construction companies.

\section{Construction Safety Issues in the Egyptian Construction Industry}

In comparison of the safety approaches, the results demonstrated noticeable differences between the work models used in construction firms in Egypt as compared to those in the EU and the United States. The major perceived deficiencies in the Egyptian construction corporations include:

- Safety plans adopted by contractors in Egypt were not well formalized compared to those applied by their American counterparts. Only few companies out of the surveyed sample had accident records broken down by projects and only a few provided workers with formal safety orientations.

- It was obvious, from the onset of our scrutiny of the safety laws implemented in Egypt that a study should be recommended to address a much needed reform in the way the employer contributes to social insurance; in order to link accident insurance rates to the contractor's safety performance. This is necessary to provide a strong incentive to the safety management and administration of safety plans. Currently, a formal safety program implementation will not reduce the actual insurance costs. Contractors are charged at the same percentage rate, regardless of their safety record. This situation eliminates the motivation to implement a formal rigorous safety program.

- Twenty percent of the firms provided workers with informal orientation. Another $20 \%$ of the firms gave workers formal orientation. In the last case, the foreman, not the safety manager, was responsible for new workers' formal orientation. The remaining percentage didn't provide the workers with any orientation.

- Ninety percent answered positively to the inquisition to determine whether the company had a full-time safety director at the corporate level. Respondents were further asked to identify the designated managerial position that the safety director reports to.

- As for the funding and whether an allocation is routinely made in the budget for the various aspects of the safety program, a statistically negligible number of contractors responded positively. Out of these negligible responses, a range between $0.5 \%-1 \%$ of the annual dollar volume was spent on safety. It is then safe to assume that this line item is absent from their annual budget.

- An assessment related to the involvement at the presidential level was investigated, asking contactors to identify the managerial level that reviews accident reports at the corporate office. Responses revealed that in none of the surveyed firms, accident reports were reviewed by the president.

\section{Goals of Educational Programs}

This study confirmed the positive impact of implementing educational programs on work hazards. Such educational programs may include:

- Safety program: The safety program should include education of employers and workers about work-related hazards, placement of workers in jobs that do not jeopardize their safety or health, early detection of adverse health effects, and referral of workers for diagnosis and treatment. The occurrence of disease or other work-related adverse health effects should prompt immediate evaluation of primary preventive measures (e.g., industrial hygiene monitoring, engi- 
neering controls, and personal protective equipment).

- Medical monitoring program: A medical monitoring program is intended to supplement, not replace, such measures. To place workers effectively and to detect and control work-related health effects, medical evaluations must be performed 1) before job placement, 2) periodically during employment, and 3) at time of job transfer or termination [11].

- Conducting daily orientations and meetings before work: That is considered to be a repeated message about safety.

- Ensuring that every employee shows full understanding of the work policies and consequences of violations.

\section{Findings, Results, and Discussions}

\subsection{Findings}

There are many hazards and accidents that occur in construction industry workplaces. However, knowing the causes of an accident is useful to develop and implement a program for prevention accidents in the future. Figure 1 shows that falls from elevation generally represent the leading cause of a death due to construction related activity, representing about $33 \%$ of all construction fatalities. Getting struck by some object, caught in-between two objects, and electrical shock are also leading factors of construction fatalities. Together, these make up about $90 \%$ of all construction fatalities. It is important to note that over the last few years, these numbers have statistically declined or increased in a linear, proportional fashion. The Occupational Safety and Health Administration (OSHA) Statistics show that about $90 \%$ of the fatalities occur from four types of injuries. These injuries may be from falls from height such as scaffolding or ladders, being struck by objects, being caught between objects, and electrocution. There are other common hazards in construction sites that may include power tools and equipment, heavy machinery, excavations, and confined spaces. Figure 1 is a graphical representation of the major causes of construction related deaths in the USA.

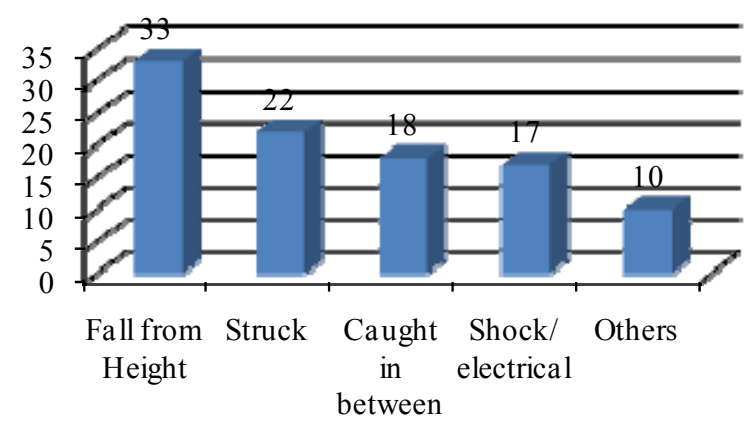

Figure. 1 OSHA Statistics of four top causes of death in the USA.
Also, in the European Union, the fatal accident rate is nearly 13 workers per 100,000 as against 5 per 100,000 for the all sector average (Eurostat Construction Accident statistics) and (Construction Safety Management Systems). Also there is 15 out of every 100 full-time construction workers in Europe suffer a lost-time injury annually, which is more than other industries. Sprains and muscle strains accounts for 38\%. Some estimates indicate that 50,000 workers a year (137 each day) die from disease contracted on the job in all industries. Falls remain the number one killer in construction work. Falls cause 100,000 injuries and up to 200 deaths annually in Europe's construction. Therefore, $85 \%$ of fall injuries results in lost time that costs employers $\$ 2$ billion annually in worker compensation and productivity. The construction industry accidents result in lost fingers, herniated discs, smashed toes, crushed bodies by pieces of machinery, injuries from bucket swings, iron splinters, and suffocation in confined spaces.

Since fall from height constitute a $33 \%$ of all construction fatalities, the fall protection requirements for residential construction are set out in Subpart M at 29 CFR 1926.501(b)(13). The standard does not mention personal fall restraint systems and OSHA has previously stated that it accepts a properly utilized fall restraint system in lieu of a personal fall arrest system when the restraint system is rigged in such a way that the worker cannot get to the fall hazard. (OSHA notes that fall protection requirements for residential construction work performed on scaffolds, ladders, and aerial lifts are in Subpart L, Subpart X, and 29 CFR 1926.453 respectively, not 29 CFR 1926.501(b)(13). The International Labour Organization (ILO) reported that about 1.9 to 2.3 million people are killed by their work every year and 25 million people have workplace injuries, causing them to take time off. In developing countries, the death rate in construction is even higher (about 10 times that in industrialized country).

In many work sites in Egypt that the team investigated, no training programs for workers existed; therefore, no orientation for new staff or workers has been conducted. Hazards were not pointed out and safety meetings were not held. In addition, there was lack of medical facilities and standard sanitation in the project worksite. Generally there was under reporting of worksite injuries. Usually, the injured employee may receive first aid management while there is unavailable specialized medical care. However, fatal accidents that resulted in death are reported because of financial expenses and litigations involved. Inspection schedules and maintaining the equipment were often not fulfilled except after an accident happened. Young workers did not usually comply with safe work procedures. This may be because of lack of experience or their negligence in wearing personal pro- 
tective equipment. The published results [12,13] of studying the Safety Programs in Large-Size Construction Firms Operating in Egypt revealed that the safety programs applied by large-size contractors operating in Egypt are less formal than those applied by their American counterparts. Only a few companies out of the surveyed sample had accident records broken down by projects; provided workers with formal safety orientation, and trained safety personnel in first-aid. Figure 2 shows the Contribution of safety practices to the safety program.

A challenge within the construction industry when dealing with occupational health is the recording and reporting of health hazards and illnesses caused. OSHA recordable criteria include death, loss of consciousness, days away from work, restricted work, job transfers, factors affecting routine job functions, "Significant" injuries or illnesses diagnosed by physician such as cancer, asbestosis, fractures or punctured eardrums, and medical treatment beyond first aid. Many illnesses do not require immediate, or any, medical attention; therefore they do not cause an "incident" and are not reported. There is a consistent trend among construction workers of "willful underreporting" of occupational health problems in fear of missing work, or a promotion in a competitive market [14]. Underreporting of illness means that preventative measures are not taken and early treatment is not conducted to improve the conditions.

\subsection{Results and Discussions}

Table 1 shows the type of injuries recorded at the egyptian work site reflecting that negligence in wearing protective equipment is the most leading cause of workplace

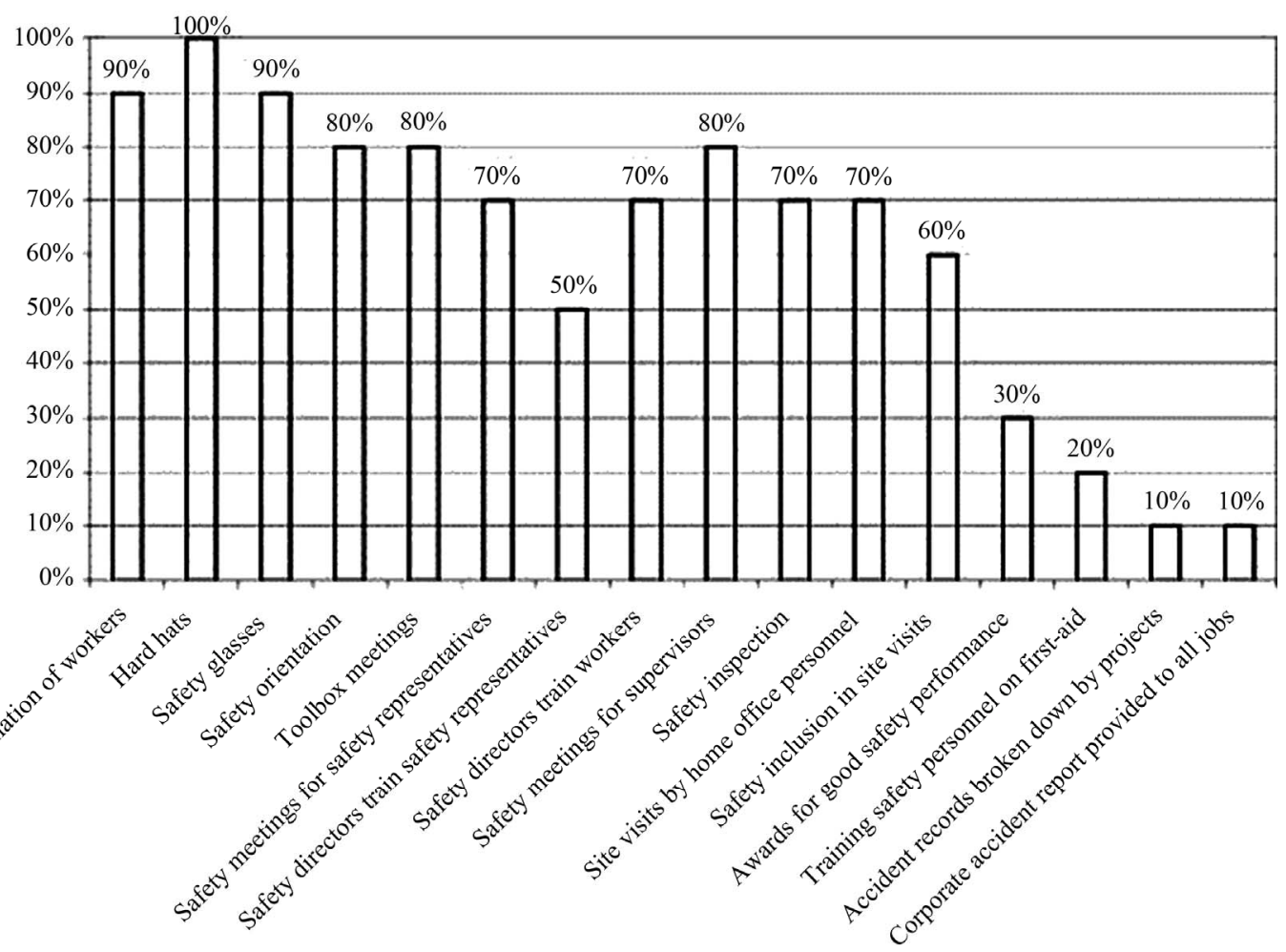

Figure 2. Contribution of safety practices to safety programs (courtesy of Amr et al., 2008).

Table 1.Types of recordable injuries \& lost work days in Egypt's company in 2008.

\begin{tabular}{ccc}
\hline Types of Injury & Egypt's Modern Construction Co. & DART \& Total lost days \\
\hline Fall from height & 3 & 63 \\
Sprain Ankles & 8 & 80 \\
Shoulder pain & 8 & 48 \\
Back pain & 6 & 126 \\
Nail in feet & 12 & 0 \\
Foreign body in eye & 14 & 14 \\
Total & 51 & 331 \\
\hline
\end{tabular}


injuries (12 cases of nail in feet and 14 cases of foreign body in eyes). Also, manual handling, awkward postures and repetitive movements are core determinants of physical load in the studied construction worksite, as reflected by high prevalence of shoulder ( 8 cases) and back ( 6 cases) pain, and sprain in ankles ( 8 cases). Ergonomic work place interventions may offer the solution of such problem. Another finding was that the majority of those musculoskeletal injuries were more occurring among young employees with age less than 30 years. However, older employees experienced longer days away from work. This is consistent with the results published by a study [15] that explained injuries among elder employees by sensory and cognitive disabilities. While there is an association between cognitive function and physical function in healthy older adults [16], cognitive impairment is associated with depression and dementia and with an increased risk of falling [17]. On the other hand, limited job knowledge and experience for young adults (aged less than 30 years old) increase their risk of injury.

Figure 3 shows a comparison between average recordable incident rates for the studied Egyptian Construction Company, US Construction Company, and general industry for 2004 through 2008. It is worth noting that the data for the Egyptian company were not avail- able for 2004 and only the data from 2005 to 2008 were recorded.

As shown in Figure 3, there is a noticed decrease in the incidence rate of recordable injuries in the construction worksite over the last five years; yet safety programs have to be fully developed and implemented by companies. This study shows that there are great benefits realized from these programs in reducing recordable incidents. Figure 4 clearly shows a comparison between recordable injuries in the Egyptian and the US construction companies.

Figures $\mathbf{4}$ and $\mathbf{5}$ show the impact of implementation of safety programs on significantly reducing recordable injuries at work site. That greatly emphasizes the importance of the benefits of enhancing the safety culture at job sites. The Egyptian construction company made preventive efforts on reducing recordable safety occurrences, and greatly reduced their incidence. The US Company's implementation of safety programs, including "The Safety and Health Achievement Recognition Program"(SHARP), improved the safety of the workers and reduced OSHA recordable injuries, as shown in Figures 4 and 5.

As shown in Figure 6, there is a decrease in DART rate due to the implementation of safety programs on recordable Injuries.

\section{Average Recordable Incident Rates}

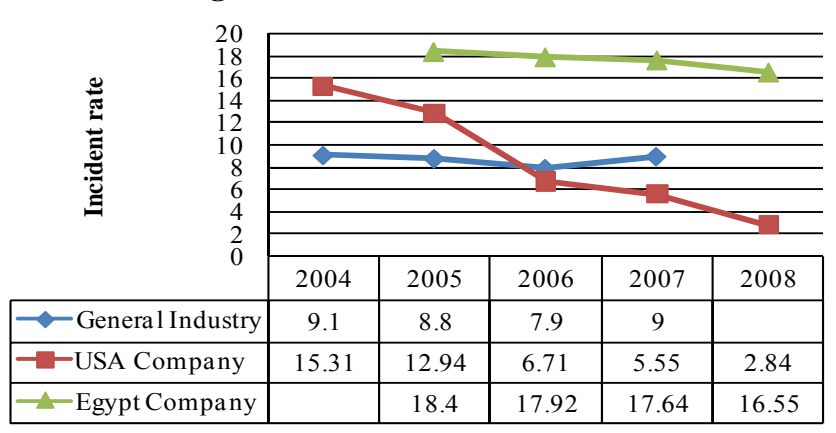

Figure 3. Comparison between average recordable incident rates.

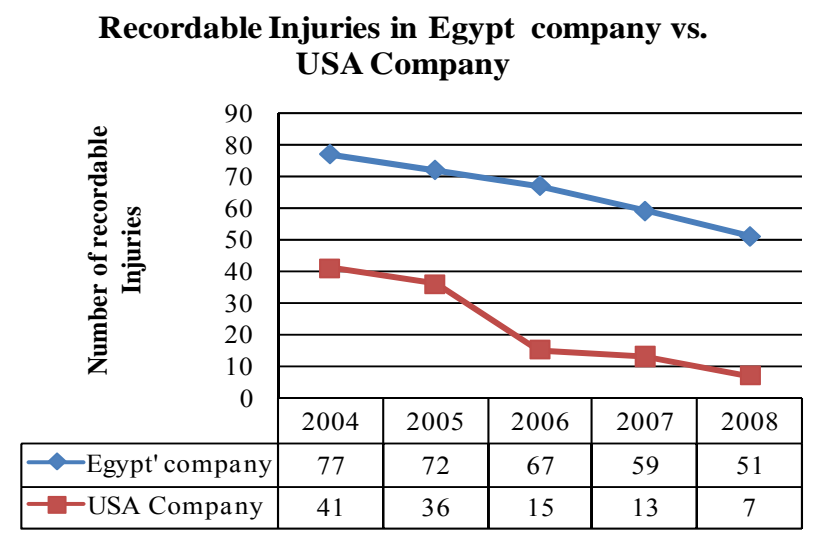

Figure 4. Recordable injuries in Egypt construction company vs. Gate Company. 
Impact of Implementation of Safety Programs on recordable Injuries

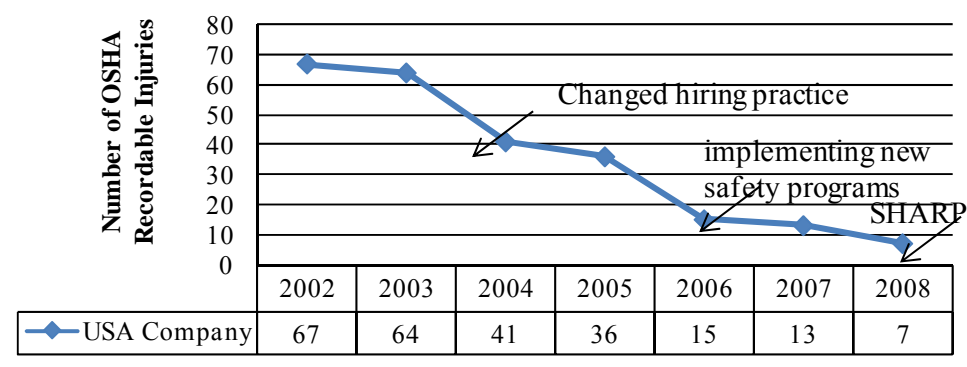

Figure 5. Number of recordable OSHA injuries in the USA company.

Average DART Rate in USA company \& General industry

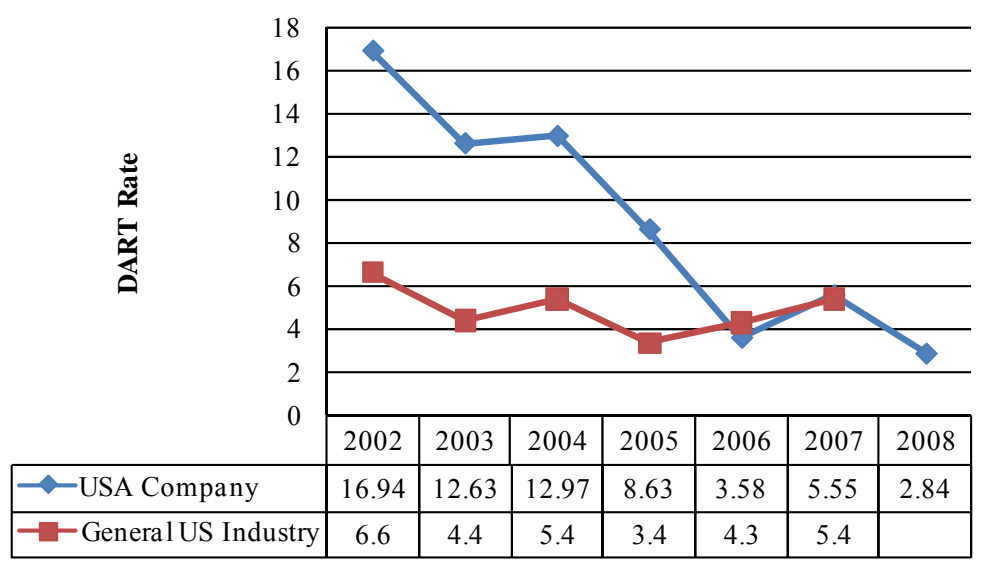

Figure 6. Comparison of average DART rates in USA company and General industry.

\section{Conclusions and Lessons Learned}

In general, large construction firms appear to have good safety records. Also, the US firms tend to have better safety record than their counterpart in Egypt, as expected. Yet, there is a positive trend of improvement in the studied firms in both countries. Primary to the safety mission, training appears to be among the stronger components of a safety program.

- There are many benefits to focusing on occupational health and wellness of the workers.

- The instructor should have access to illustrated booklets that cover specific OSHA code segments, including everything from confined spaces and fall protection to welding sites, fire safety, and material handling.

- Frequent orientation seminars are important and workers are encouraged to report injuries, accidents, and near-misses. Workers should always be provided with a safety phone number for emergency situations, and continuous advice.

- Timely management feedback on safety concerns should be implemented.
- Implementing new safety programs including "The Safety and Health Achievement Recognition Program"-(SHARP) reduces significantly the recordable injuries.

- This study emphasizes the importance of providing effective fall protection safeguards and training to workers. The study encourages refusing site access to badgeless workers, and those without reflective safety vests, hardhats, and protective eyewear.

- Incentives can result in better safety performances. Also, site specific safety programs, raises based on safety performance, creating personal safety records, worker safety perception surveys, and documenting near-misses can result in better safety records.

- Training appears to be among the stronger components of a safety program and an OSHA 10-hour training course is highly recommended for job site workers. Enforce 10 OSHA hours training or undergo mandatory onsite training. Also, conduct daily training to reach individual workers every day.

- Knowing that an unhealthy worker or risky work environment, often leads to low company productivity, 
lost wages for employees.

- Safety consciousness is a vital professional qualification for construction worker.

- Fatigue and weariness should be avoided since they compromise safety.

- Maintain zero tolerance for unsafe construction practices by enforcing strict discipline against violators.

- Demonstrate commitment to safety culture through managers, supervisors, and workers thus creating a safer working environment.

- The laws in Egypt need to be addressed to recommend changes in the insurance laws to reflect the company's record of safety. That should provide an incentive for construction companies to enhance their safety programs.

\section{REFERENCES}

[1] OSHA - Occupational Safety and Health Administration, "OSHA Forms for Recording Work-Related Injuries and Illnesses,” 2003.

http://www.osha.gov/recordkeeping/new-osha300form1-1 -04.pdf

[2] OSHA - Occupational Safety and Health Administration. "OSHA Regulations (Standards-29 CFR)," 2006.

[3] OSHA-Occupational Safety and Health Administration, "Occupational Safety and Health Act of 1970," 2009.

[4] BLS-U.S. Bureau of Labor Statistics, "Workplace Injuries and Illnesses in 2007," 2008. http://www.bls.gov/iif/oshwc/osh/os/osnr0030.pdf

[5] BLS-U.S. Bureau of Labor Statistics, "Survey of Occupational Injury and Illness-2008,” 2009.

http://www.bls.gov/iif/oshsum.htm

[6] F. Murie, "Preventing Injuries and Ill-Health in the Construction Industry, Health and Safety at Work: A Trade Union Priority," Labour Education 2002/1, No. 126, 2002.

[7] NORA - National Occupational Research Agenda, "National Construction Agenda for Occupational Safety and Health Research and Practice in the U.S. Construction Sector," 2008.
http://www.cdc.gov/niosh/NORA/comment/agendas/cons truction/pdfs/ConstOct2008.pdf

[8] T. S. Abdel Hamid and J. G. Everett, "Identifying Root Causes of Construction Accidents," Journal of Construction Engineering and Management, Vol. 126, No. 1, 2000, pp. 52-60. doi:10.1061/(ASCE)0733-9364(2000)126:1(52)

[9] D. H. Baldev, "Design of a Construction Safety Training System using Contextual Design Methodology," Master's Thesis, Virginia Polytechnic Institute and State University, Blacksburg, 2006.

[10] P. Hughes and E. Ferrett, "Introduction to Health and Safety in Construction," 2nd Edition, Elsevier Ltd., Oxford, 2007.

[11] OSHA - Occupational Safety and Health Administration, "Occupational Safety and Health Guidelines," 2009. http://www.osha.gov/SLTC/healthguidelines/index.html

[12] A. A. G. Hassanein and R. S. Hanna, "Safety Programs in Large-Size Construction Firms Operating in Egypt," Journal of SH\&E Research, Vol. 4, No. 1, 2007, pp. 1-31.

[13] A. A. G. Hassanein and R. S. Hanna, "Safety Performance in the Egyptian Construction Industry," Journal of Construction Engineering and Management, Vol. 134, No. 6, 2008, pp. 451-455. doi:10.1061/(ASCE)0733-9364(2008)134:6(451)

[14] J. P. Leigh, S. Markowitz, M. Fahs and P. Landrigan, "Costs of Occupational Injuries and Illnesses," University of Michigan, Ann Arbor, 2000.

[15] N. Chau, A. Bhattacherjee and B. M. Kunar, "Relationship between Job, Lifestyle, Age and Occupational Injuries," Occupational Medicine, Vol. 59, No. 2, 2009, pp. 114-119. doi:10.1093/occmed/kqp002

[16] G. C. Gauchard, D. Deviterne and F. Guillemin, "Prevalence of Sensorial and Cognitive Disabilities and Falls, and Their Relationships: A Community-Based Study,' Neuroepidemiology, Vol. 26, No. 2, 2006, pp. 108-118.

[17] C. Rosano, E. M. Simonsick and T. B. Harris, "Association between Physical and Cognitive Function in Healthy Elderly: The Health, Aging and Body Composition Study," Neuroepidemiology, Vol. 24, No. 1-2, 2005, pp. 8-14. doi:10.1159/000081043 